subsequently with CNK. At the moment of CNK introduction and at their latest visit while on CNK, patients were asked to fill a questionnaire investigating their experience with either ANK or CNK. The questionnaire included 4 different domains that were assessed through means of Visual Analogue Scales (VAS) ranging from 0 to 10 . The following domains were considered: injection-related pain, satisfaction with the frequency of administration, concern for drug-related adverse events including infections, and overall drug tolerability. VAS values for each of the domains were compared between ANK and CNK with Mann-Whitney U test.

Results: Ten AOSD patients treated with both ANK and CNK were included in the analysis. Disease characteristics are shown in Table 1 below. ANK was discontinued due to inefficacy in 6 patients and due to adverse reactions in 4 . As shown in Figure 1, CNK overall tolerability significantly exceeded ANK one $(p=0.021)$. Only 1 AOSD patient reported a greater tolerability with ANK compared to CNK because she felt more comfortable with pre-filled syringes. The reasons for higher CNK tolerability were the lower pain at injection site (median 1, IQR 1-1 vs median 4.5, IQR 1.25-7; $p=0.015$ ) and the greater level of satisfaction with the frequency of administration (median 10, IQR 9.25-10 vs median 3.5, IQR 2.25-4.75, $p<0.001$ ). The concern for drug-related adverse events was not significantly different between ANK and CNK $(p=0.549)$ and overall low among AOSD patients. CNK led to a satisfying control of systemic inflammation and steroid-sparing effect in most of patients and it was discontinued in only one case (Patient 5), due to ineffective disease control.
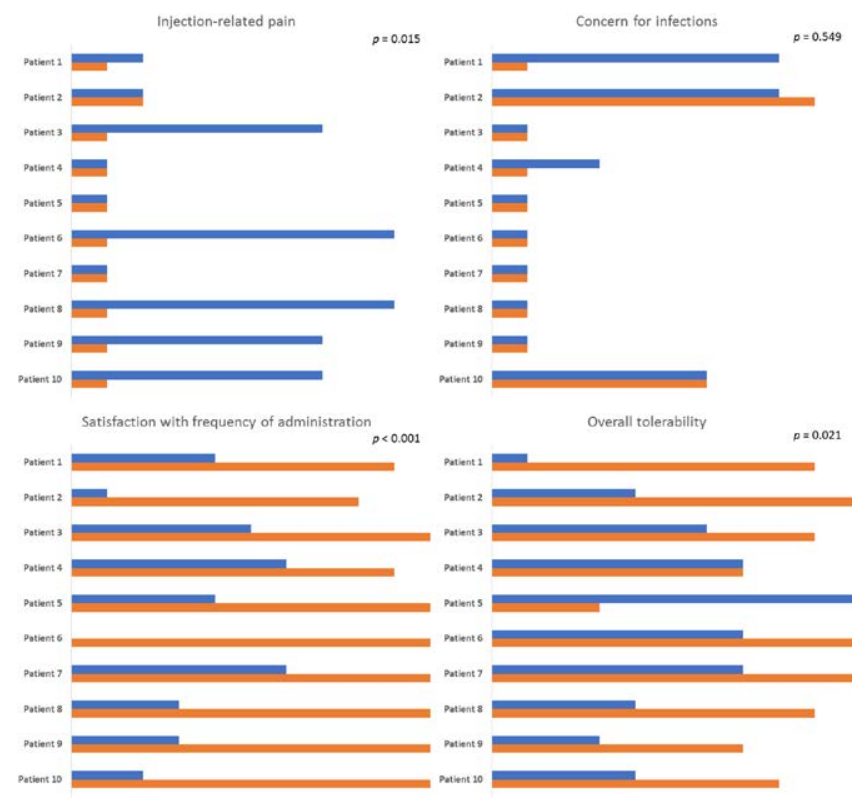

Figure 1. Comparative tolerability of anakinra (blue line) and canakinumab (orange line) in our study population, expressed as Visual Analogue Scale values (range from 1 to 10). Reported $p$-values refer to the comparative analysis between the two drugs.
Conclusion: In our cohort of AOSD patients, CNK proved not only to be effective in controlling systemic inflammation in ANK refractory cases, but its use was associated with greater patients' satisfaction and tolerability.

REFERENCES:

[1] Kluetz et al, Value Health, 2018;21(6):742-747.

Disclosure of Interests: Nicola Farina: None declared, Corrado Campochiaro Speakers bureau: Novartis, Pfizer, Roche, GSK, SOBI, Alessandro Tomelleri: None declared, Giacomo De Luca Speakers bureau: SOBI, Novartis, Celgene, MSD, Pfizer, Giulio Cavalli Speakers bureau: SOBI, Elena Baldissera Speakers bureau: Novartis, Pfizer, Roche, Alpha Sigma, Sanofi, Lorenzo Dagna Speakers bureau: Abbvie, Amgen, Biogen, BMS, Celltrion, Novartis, Pfizer, Roche, SG, SOBI, Celgene Janssen, MSD, MP

DOI: 10.1136/annrheumdis-2021-eular.1751

\section{POS1348 \\ MORTALITY AND ITS RELATED FACTORS IN PATIENTS WITH IGG4-RELATED DISEASE: A JAPANESE SINGLE-CENTER STUDY}

1. Mizushima ${ }^{1}$, H. Kawahara ${ }^{1}$, T. Yoshinobu', S. Shin ${ }^{1}$, R. Hoshiba ${ }^{1}$,

R. Nishioka ${ }^{1}$, T. Zoshima ${ }^{1}$, S. Hara' ${ }^{1}$, Y. Suzuki ${ }^{1}$, K. Ito ${ }^{1}$, M. Kawano ${ }^{1} .{ }^{1}$ Kanazawa University Hospital, Rheumatology, Kanazawa, Japan

Background: In recent years, IgG4-related disease (IgG4-RD) has become a widely recognized disorder. However, mortality and its related factors in this disease are not well known.

Objectives: This study aimed to clarify mortality and its related factors in patients with IgG4-RD.

Methods: We retrospectively reviewed the medical records of patients with IgG4-RD diagnosed by experts based on fulfillment of the Japanese comprehensive diagnostic criteria and/or the 2019 ACR/EULAR classification criteria for IgG4-RD at a single center in Japan. Using the collected data, we calculated the crude mortality rate and the standardized mortality ratio (SMR) using national Japan mortality statistics and investigated the cause of death. We performed Cox regression analyses to assess mortality-related factors.

Results: A total of 179 patients with IgG4-RD were included: 124 were male (69.3\%); the median age was 68 years (interquartile range [IQR] 60-75 years); and the median follow-up from diagnosis was 47 months (IQR 17-84). Ten patients $(5.6 \%)$ in our cohort died during the follow-up period. Five died of malignancy, one of respiratory failure, two of infectious pneumonia, one of sudden cardiac event, and one of suspected aortic aneurysmal rupture. The crude mortality rate was 11.1 per 1,000 person-years. According to national Japan mortality statistics, 11.6 age- and sex-matched deaths were expected to occur within the follow-up period, resulting in a SMR of 0.86 (95\% confidence interval [CI] 0.41-1.59). Univariate Cox regression analyses indicated that the number of affected organs at diagnosis (hazard ratio [HR] 1.45, 95\% Cl 1.02-2.05), serum creatinine levels at diagnosis ( $\mathrm{HR} 1.82,95 \% \mathrm{Cl} 1.06-3.12)$, and the presence of malignancy during the clinical course (HR $3.93,95 \% \mathrm{Cl} 1.10-14.02)$ had a significant impact on the time to death, whereas the other factors including age at diagnosis and serum C-reactive protein and IgG4 levels at diagnosis did not.

Conclusion: Our findings suggest that the mortality rate of patients with IgG4-RD does not significantly differ from that of the Japanese general

Table 1. Clinical and treatment characteristics of study population.

\begin{tabular}{|c|c|c|c|c|c|c|c|}
\hline & Sex and age (years) & $\begin{array}{l}\text { Disease duration } \\
\text { (months) }\end{array}$ & Disease manifestations & $\begin{array}{l}\text { Sequential biologic } \\
\text { therapies }\end{array}$ & $\begin{array}{l}\text { Steroid dosage varia- } \\
\text { tion (mg/day) }\end{array}$ & $\begin{array}{l}\text { ESR variation }(\mathrm{mm} / \\
\text { hour) }\end{array}$ & $\begin{array}{l}\text { CRP varia- } \\
\text { tion (mg/L)" }\end{array}$ \\
\hline Patient 1 & Male, 70 & 22 & $\begin{array}{l}\text { Arthritis, Fever } \\
\text { Rash }\end{array}$ & TCZ, ANK, CNK & $25 \rightarrow 5$ & $120 \rightarrow 13$ & $55 \rightarrow 1$ \\
\hline Patient 2 & Female, 72 & 11 & $\begin{array}{c}\text { Arthritis, Fever } \\
\text { Rash }\end{array}$ & TCZ, ANK, CNK & $5 \rightarrow 0$ & $60 \rightarrow 24$ & $265 \rightarrow 6$ \\
\hline Patient 3 & Male, 32 & 48 & Fever, Rash & ANK, CNK & $15 \rightarrow 0$ & $29 \rightarrow 11$ & $27 \rightarrow 1$ \\
\hline Patient 4 & Male, 71 & 12 & Fever & ANK, CNK & $20 \rightarrow 5$ & $120 \rightarrow 2$ & $180 \rightarrow 1$ \\
\hline Patient 5 & Female, 58 & 72 & Fever & ANK, CNK & $0 \rightarrow 5$ & $27 \rightarrow 36$ & $5 \rightarrow 83$ \\
\hline Patient 6 & Female, 48 & 5 & $\begin{array}{l}\text { Arthritis, Fever } \\
\text { Rash }\end{array}$ & ANK, CNK & $10 \rightarrow 0$ & $8 \rightarrow 10$ & $2 \rightarrow 1$ \\
\hline Patient 7 & Female, 74 & 180 & Arthritis, Fever, Rash, MAS & ETN, ANK, CNK & $5 \rightarrow 5$ & $33 \rightarrow 1$ & $1 \rightarrow 25$ \\
\hline Patient 8 & Male, 78 & 180 & $\begin{array}{c}\text { Arthritis, Fever } \\
\text { Rash, Myocarditis } \\
\text { Pleuropericarditis }\end{array}$ & ANK, CNK & $40 \rightarrow 20$ & $46 \rightarrow 10$ & $58 \rightarrow 40$ \\
\hline Patient 9 & Male, 22 & 144 & Arthritis, Fever & ETN, TCZ, ANK, CNK & $20 \rightarrow 10$ & $86 \rightarrow 94$ & $135 \rightarrow 1$ \\
\hline Patient 10 & Male, 43 & 24 & $\begin{array}{c}\text { Arthritis, Fever } \\
\text { Rash }\end{array}$ & ANK, CNK & $15 \rightarrow 5$ & $15 \rightarrow 10$ & $4 \rightarrow 1$ \\
\hline
\end{tabular}

Before and after canakinumab start.

ANK, anakinra; CNK, canakinumab; CRP, C-reactive protein; ESR, erythrocyte sedimentation rate; ETN, etanercept; MAS, macrophage activation syndrome; MTX, methotrexate; PDN, prednisone; TCZ, tocilizumab. 
population. Multi-organ involvement and renal dysfunction at diagnosis as well as malignancy during the clinical course may be associated with higher mortality. An appropriate clinical evaluation for the early detection of these risk factors is required at first diagnosis and during long-term follow-up.

Disclosure of Interests: None declared

DOI: 10.1136/annrheumdis-2021-eular.1798

\section{POS1349 THE JOINT INVOLVEMENT IN ADULT ONSET STILL'S DISEASE IS CHARACTERISED BY A PECULIAR MAGNETIC RESONANCE IMAGING AND A SPECIFIC TRANSCRIPTOMIC PROFILE}

P. Ruscitti ${ }^{1}$, A. Barile ${ }^{1}$, O. Berardicurti ${ }^{1}$, S. lafrate ${ }^{1}$, P. DI Benedetto ${ }^{1}$, A. Vitale ${ }^{2}$, F. Caso ${ }^{3}$, L. Costa ${ }^{3}$, F. Bruno ${ }^{1}$, F. Ursini ${ }^{4}$, L. Navarini ${ }^{5}$, F. Sensini ${ }^{1}$, R. Scarpa ${ }^{3}$, B. Frediani ${ }^{2}$, L. Cantarini ${ }^{2}$, C. Masciocchi $^{1}$, R. Giacomelli ${ }^{5}$, P. Cipriani ${ }^{1}$. 'University of LAquila, Department of Biotechnological and Applied Clinical Sciences, L'Aquila, Italy; ${ }^{2}$ University of Siena, Research Center of Systemic Autoinflammatory Diseases and Behçet's Disease Clinic, Department of Medical Sciences, Surgery and Neurosciences, Siena, Italy; ${ }^{3}$ University Federico II of Naples, Department of Clinical Medicine and Surgery, School of Medicine, Naples, Italy; ${ }^{4}$ RRCS Istituto Ortopedico Rizzoli, Bologna, Department of Biomedical and Neuromotor Sciences, University of Bologna, Bologna, Italy; ${ }^{5}$ University of Campus Bio-Medico of Rome, Unit of Allergology, Immunology, Rheumatology, Department of Medicine, Rome, Italy

Background: Adult onset Still's disease (AOSD) is a rare systemic autoinflammatory disease and joint involvement is one of its clinical manifestations [1]. Arthritis, either oligoarthritis or bilateral symmetrical rheumatoid arthritis-like polyarthritis, is another common clinical feature of AOSD, with a migrating pattern at the beginning and becoming stable over the time [1].

Objectives: The aims of the study were to assess joint involvement in AOSD by using magnetic resonance imaging (MRI), to describe main patterns of involvement, and associated clinical characteristics, and to evaluate the global transcriptomic profile of synovial tissues in AOSD to elucidate possible pathogenic pathways involved with.

Methods: AOSD patients, who underwent to magnetic resonance imaging (MRI) exam on joints, were assessed to describe patterns of joint involvement and associated clinical characteristics. Some synovial tissues were collected for RNA-sequencing purposes.

Results: In this study, 31 patients with AOSD (mean age $42.3 \pm 15.2$ years, $54.8 \%$ male gender), who underwent to at least one MRI exam on joints, were assessed. The most common MRI finding was the presence of synovitis on $60.5 \%$, mainly in peripheral affected joints. MRI revealed a mild to moderate proliferative synovitis, as thickening of the synovial membrane, with low to intermediate signal intensity on T1-weighted images and intermediate to high signal intensity on T2-fat saturated weighted and STIR images, suggesting the presence of a hyperplastic than of a hypertrophied synovial tissue. Bone oedema and bone erosions were reported on $34.9 \%$ and $25.6 \%$ MRI exams, respectively. In all patients but one, bone erosions were synchronous with bone oedema, overlapping completely the locations. Assessing clinical characteristics in patients with MRI-erosions, a higher prevalence of splenomegaly, a more frequent chronic disease course, lower levels of erythrocyte sedimentation rate and ferritin was observed.

Assessing the synovial tissues of some AOSD patients, a moderate perivascular mononuclear infiltrate in the sub-lining stroma of hip synovial tissues was observed, whereas the lining cells were relatively unremarkable. In addition, interleukin (IL)-1ß, IL-6, TNF, and heavy ferritin subunit (FeH) were found on AOSD synovial tissues.

An RNA-sequencing analysis assessed the global transcriptomic profile of synovial tissues on AOSD patients and matched-controls. Assessing IL-1 pathway, we found an increased expression of il1a, il1b, il1 rap, il1r1, il18r1, and $1 / 18 \mathrm{bp}$ on AOSD tissues when compared with controls. In IL-6 pathway, we found an increased expression of il6 and il6st/gp130 on AOSD synovial tissues whereas an increased expression of il6r was shown on the controls. Among genes involved in TNF pathway, tnf, traf1, traf2, tnfaip3 and tnfrsf1a resulted to be more expressed in AOSD synovial tissues than in controls. Finally, fth1 and ftl were more expressed in AOSD patients than controls, when we explored the iron uptake and transport pathway.

Conclusion: A peculiar MRI pattern of joint involvement in AOSD was reported; the most common finding was the presence of synovitis, characterised by intermediate to high signal intensity on T2-fat-saturated weighted and STIR images. Bone erosions and bone oedema were also observed. This MRI pattern was associated with a hyper-activation of IL-1, IL-6, and TNF pathways together with a hyper-expression of ferritin genes on AOSD synovial tissues.

\section{REFERENCES:}

[1] Giacomelli R, Ruscitti P, Shoenfeld Y. A comprehensive review on adult onset Still's disease. J Autoimmun. 2018;93:24-36.

Disclosure of Interests: None declared

DOI: 10.1136/annrheumdis-2021-eular.1844

\section{POS1350 FAMILIAL MEDITERRANEAN FEVER DURING PREGNANCY: A 26 CASE SERIES WITH 38 PREGNANCIES}

Y. Karabulut ${ }^{1} .{ }^{1}$ Private Yıldırım Doruk Hospital, Division of Rheumatology, Bursa, Turkey

Background: Familial Mediterranean Fever (FMF) is an autoinflammatory disease characterized by recurrent attacks with autosomal recessive inheritance. FMF usually occurs in young age; most patients (90\%) begin to suffer from their first attack before 20 years of age. Pregnancy can occur frequently during the course of the disease, as women of reproductive age are affected by the disease.

Objectives: In this study, it was aimed to retrospectively examine the demographic, genetic, and clinical features (frequency of attacks during pregnancy, duration of attacks, treatment of attacks) of 26 cases who were followed up with a diagnosis of FMF in the last five years and experienced pregnancy.

Methods: A total of twenty-six female FMF cases experienced pregnancy and diagnosed or followed up in our rheumatology center between 2015-2020 were included in the study. All patients were diagnosed according to Tel-Heshomer criteria. All data and follow-up visit records of the patients were retrospectively recorded by the rheumatologist. Patients were followed up by an obstetrician working in the same center during pregnancy. The demographic and genetic characteristics of the patients, the treatment used, the duration and characteristics of the attack during pregnancy, and the treatment they received during the attack were recorded retrospectively. Data processing and analysis conducted with SPSS 22 for Windows.

Results: During the follow-up period, a total of 38 pregnancies were observed in 26 female cases. When the genetic mutation tests of all patients were examined, $61 \%$ were $\mathrm{M} 694,15 \%$ were $\mathrm{V} 726,11 \%$ were $\mathrm{M} 680 \mathrm{l}$ positive and compound mutation was detected in $42 \%$ of the patients. The mean age of the patients was $30 \pm 7.8$, the disease duration was $9.8 \pm 5.4$ years, the follow-up period was $38 \pm 14$ months, the attack frequency during pregnancy was $3.6 \pm 1.7$ and the attack duration was $14 \pm 9.8$ hours. Considering the clinical features, fever was seen in $92.3 \%$, abdominal pain $96.1 \%$, chest pain $88.4 \%$, arthritis $11.5 \%$ and other symptoms seen in $26 \%$ during attacks of pregnant FMF patients. All patients used $1 \mathrm{gram}$ of colchicine regularly throughout pregnancy. Steroids were used in $11.5 \%$ of patients and non-steroid anti-inflammatory drugs in $53.8 \%$ of patients during the attack. Anakinra was used in $11.5 \%$ of the cases except for the first trimester following a written consent obtained from the patients.

In $10.5 \%$ of 38 pregnancies, spontaneous abortion was observed in the early period, $7.8 \%$ of pregnancies resulted in preterm delivery before 32 weeks. In addition, $81.5 \%$ of pregnancy completed the planned period and resulted in a healthy birth. Cesarean section was performed in 4 patients and normal delivery procedure in 27 patients. Major malformation-anomaly was not observed in any baby. When patients using colchicine (73\%) irregularly and less than 1 gram $(26.9 \%)$ before pregnancy were compared in terms of attack frequency and duration, the group using regular medication had significantly fewer and shorter attacks $(p<0.05)$

Three colchicine resistant patients with M694 homozygous mutation became pregnant under anakinra treatment. A total of five pregnancies were followed in three cases. No medication was used in these patients in the first trimester As of the second trimester, $100 \mathrm{mg} /$ day for 3 days of anakinra was administered in these patients after obtaining an informed consent. In this patient group, no obstetric problem was observed during and after pregnancy, and healthy deliveries were realized.

Conclusion: Pregnancy is common in FMF patients of reproductive age. Disease and relapse treatment during pregnancy is still a problem due to the limited number of medications that can be used for treatment. Furthe studies required to verify safety of Anakinra in refractory FMF cases. There is a need to develop options for the prevention and treatment of attacks during pregnancy.

Disclosure of Interests: None declared

DOI: 10.1136/annrheumdis-2021-eular.1878 